\title{
ALLELE FREQUENCY OF GLUTENIN SUBUNITS AND GLU-1 QUALITY SCORES IN SOME TURKISH BREAD WHEAT LANDRACES
}

\author{
Ridvan TEMIZGUL*, Mikail AKBULUT \\ Erciyes University, Faculty of Science, Department of Biology, 38039, Kayseri/ TURKEY
}

Cite this article as:

Temizgul R. Akbulut M. 2020. Allele Frequency of Glutenin subunits and Glu-1 Quality Scores in Turkish Bread Wheat Landraces. Trakya Univ J Nat Sci, 21(1): 1-11, DOI: 10.23902/trkjnat.609487

Received: 22 August 2019, Accepted: 08 November 2019, Online First: 22 November 2019, Published: 15 April 2020

Edited by:

Yerlan Turuspekov

*Corresponding Author:

Ridvan Temizgul

rtemizgul@erciyes.edu.tr

\section{ORCID ID:}

orcid.org/0000-0002-1033-7067

Key words:

Gluten

Allele frequency

Glu-1 quality score

Turkish bread wheat landraces

\begin{abstract}
There are eight centers of origin for cultivated plants and Turkey is located in the interception of two of these centers, the Near East and the Mediterranean. Therefore, Turkey is known to be the gene center for diversification and dispersion of such main cereal crops such as wheat, barley, rye and oat. This study was performed to determine glutenin gene allele frequencies and Glu-1 quality scores of 116 local wheat landraces of Turkish bread wheat. SDSPAGE and PCR were used to identify glutenin gene alleles. The results showed that the studied Turkish local wheat landraces contained a total of 19 different subunits (3 subunits in Glu-Al, 11 in $G l u-B 1$ and 5 in $G l u-D 1$ ) with 50 different combinations. The highest and the lowest allelic combinations were determined in East Anatolia and the Aegean regions, respectively. Glu-Alc (65.11\%), Glu-Blb (53.60\%) and Glu-Dla (58.30\%) were the most frequent alleles. The Glu-1 quality score was found to be 6.07 for the studied genotypes. Among the regions, the highest (7.18) and the lowest (4.80) mean Glu-1 scores were detected in Marmara and Southeastern Anatolia regions, respectively. 4 accessions (TR32846-6, TR36948-1, TR45105 and TR63536) were reported to have the highest Glu-1 quality score as 10.6 genotypes (TR45398-4, TR480253, TR33264-6, TR393-5, TR52021-3 and TR45094) had the quality score of 9. Including more new landraces may contribute to discover new Glu-1 alleles.
\end{abstract}

Özet: Kültür bitkileri için sekiz orijin merkezi vardır ve Türkiye, bu merkezlerden ikisinin, Yakın Doğu ve Akdeniz'in, kesiştiği yerdedir. Türkiye bu nedenle buğday, arpa, çavdar ve yulaf gibi ana tahıl bitkilerinin çeşitlendiği ve dağıldığ 1 gen merkezi olarak bilinir. Bu çalışmanın amacı, Türk ekmeklik buğdaylarından 116 yerel buğday ırkının glüten allel sıklığ 1 ve Glu-1 kalite skorunu belirlemektir. SDS-PAGE ve PCR, glüten allellerini tanımlamak için kullanılmıştır. İstatistiksel analizler için POPGENE 1.31 yazılımı kullanılmıştır. İncelenen Türk yerel buğday rrkları toplam 19 farklı alt birim (Glu-Al'de 3 alt birim, Glu-Bl'de 11 ve Glu-Dl'de 5 alt birim) ve 50 farklı kombinasyon içermektedir. En yüksek ve en düşük allel kombinasyonları sirasıyla Doğu Anadolu ve Ege bölgelerinde belirlenmiştir. Glu-Alc (\% 65,11), Glu-Blb (\% 53,60) ve Glu-Dla $(\% 58,30)$ en s1k görülen alleller olarak tespit edilmiștir. Glu-1 kalite skoru, Türkiye genelinde incelenen genotipler için 6,07 olarak bulunmuş̧tur. Bölgeler arasında ortalama en yüksek $(7,18)$ ve en düşük $(4,80)$ Glu-1 skorları sırasıyla Marmara ve Güneydoğu Anadolu bölgelerinde tespit edilmiştir. Çalış1lan 116 aksesyondan 4 tanesi (TR32846-6, TR36948-1, TR45105 ve TR63536) kalite skoru 10 olup en yüksek Glu- 1 kalite skoruna sahipken 6 tanesinin ise (TR45398-4, TR48025-3, TR33264-6, TR393-5, TR52021-3 ve TR45094) kalite skoru 9 olarak hesaplanmıştır. Daha fazla yerel çeşitlerin çalışmalara eklenmesi yeni Glu-1 allelerinin keşfedilmesine katkıda bulunabilir.

\section{Introduction}

Vavilov (1951) identified eight centers with gene center status that have long been used for agriculture and Turkey is located in the interception of two of these centers (Near East and the Mediterranean), making the country one of the richest in terms of plant biodiversity. Turkey is also known to be one of the gene centers for diversification and dispersion of main cereal crops such as wheat, barley, rye, and oat. This special future, in addition to being the first place where wheat was cultivated, makes the country for several wild species and landraces of wheat to be available. For instance, the first cultivated forms of diploid and tetraploid wheat originated from Karacadağ (Diyarbakır) in the Southeastern region of Turkey.

These first diploid (AA) and tetraploid genomes $(\mathrm{AABB})$ and their phylogenetic analysis indicate that they 
had originated in Southeastern Turkey (Dubcovsky \& Dvorak 2007). Cultivation shifted from here to near east about 9.000 years ago and the hexaploid wheat appeared for the first time (Feldman 2001). Cereals occupy 55\% of total agricultural and $57 \%$ of cultivated areas (21.4 million hectares) in Turkey (Grain sector report 2013). Among the cereals in Turkey, wheat is the pioneering crop with $67 \%$ share and the country supplies $3 \%$ of world wheat production every year. However, because of quality-related issues, it is still a wheat importing country (TMO 2017). Among the regions, the leading wheat producing region is Central Anatolia (32.4\%), followed by Marmara (17.1\%), Southeastern Anatolia (13.3\%), the Mediterranean (12.8\%), Black Sea (10.1\%), Aegean (7.4\%) and Eastern Anatolia (6.9\%) regions.

The quantity and composition of high molecular weight gluten subunits (HMW-GS) are important factors in determining wheat baking properties. Localization of HMW subunit genes on long arms of homologous group 1 was reported by Orth \& Bushuk (1974) and Bietzh et al. (1975). Each locus contains two linked genes called $\mathrm{x}$ and y type which are distinguished by their characteristics and molecular weights (Payne et al. 1981). However, as some of those genes are silent, the common wheat possesses 3 to $5 \mathrm{HMW}$ subunits encoded at the Glu-1 loci on the long arms of group 1 chromosomes (1A, 1B, and 1D). The contribution of $\mathrm{D}$ genome, followed by $\mathrm{B}$ genome considered to have a significant influence on good baking quality (Uthayakumaran et al. 2002). Especially, two subunits are expressed always by $G l u-D 1$, one or two by $G l u-B 1$, one or none (null allele) is expressed by $G l u-A 1$ loci. If one subunit is expressed by $G l u-A 1$ or $G l u-B 1$, this is always considered as $\mathrm{X}$-type. Rheological properties of the gluten complex are related to the presence or absence of specific subunits of these proteins. The presence of certain HMW subunits is positively correlated with good bread-making quality to determine gluten elasticity (Nakamura 2000, Shewry et al. 2003). The relationships between HMW subunits and dough elasticity were determined 40 years ago (Payne et al. 1979). Significant differences were found among protein components of wheat grain depending on cultivars, environments and their interactions (Horvat et al. 2015, Tok et al. 2011). Allelic variations in each Glu-1 loci were reported in bread wheat genotypes (Lawrence \& Shepherd 1980, Payne \& Lawrence 1983) and an enumeration system was developed to define different allelic subunits. The definition of HMW subunits coded by Glu-Dl and GluAl was described by Payne et al. (1983) and Lafiandra et al. (1997).

Subunits of gluten proteins can be identified by Sodium Dodecyl Sulphate Polyacrylamide Gel Electrophoresis (SDS-PAGE) analysis. Considering the fact that chains of gluten polymers are stabilized by disulfide bonds, using reducing agents such as DTT and mercaptoethanol in SDS-PAGE makes the identification of gluten subunits easier.

Although Turkey comes third in the production of wheat in the world, quality problems still persist. This study was performed to determine the composition and occurrence frequency of HMW glutenin subunits and the potential of the end-use quality in Turkish wheat landraces to be used in future breeding programs. Analysis of broad collections of landraces enabled the definition of rare alleles on Glu-1 loci.

Table 1. The wheat specimens studied with their respective distribution regions. All specimens were given using their gen bank accession numbers.

\begin{tabular}{|c|c|c|}
\hline Regions & Wheats (with gen bank accession numbers) & $\begin{array}{c}\text { Total } \\
\text { Number }\end{array}$ \\
\hline $\begin{array}{l}\text { Central } \\
\text { Anatolia }\end{array}$ & $\begin{array}{l}\text { TR45324-2, TR48061-4, TR53299-1, TR53343-5, TR55002-3, TR46889-2, TR45094, } \\
\text { TR47944, TR63316-4, TR35409-1, TR52021-6, TR52021-3, TR35408-4, TR35147-4, } \\
\text { TR45303-4, TR45306-2, TR45306-5, TR45308-2, TR53862-5, TR55153-4, TR55164-1, } \\
\text { TR55180-1, TR57999-2, TR57999-6, TR32034, TR63536, TR63538 }\end{array}$ & 27 \\
\hline Aegean & $\begin{array}{l}\text { TR52860-3, TR52860-6, TR52865-1, TR55127-6, TR55140-1, TR55144-1, TR55174-1, } \\
\text { TR55201-4, TR52784, TR52873, TR56099 }\end{array}$ & 11 \\
\hline Marmara & $\begin{array}{l}\text { TR33264-6, TR51937-1, TR51937-2, TR33500-4, TR33500-6, TR38316-4, TR52645, } \\
\text { TR52669, TR51937, TR44365, TR45080, TR26746 }\end{array}$ & 12 \\
\hline Mediterranean & $\begin{array}{l}\text { TR46804, TR52824, TR55316, TR55110, TR37492-1, TR37492-2, TR37492-4, TR32009-5, } \\
\text { 393-5, TR26233, TR62808 }\end{array}$ & 11 \\
\hline Black Sea & $\begin{array}{l}\text { TR14861-5, TR44487-4, TR46873-4, TR44365-4, TR44365-5, TR44984-5, TR32125-3, } \\
\text { TR37234-1, TR45105, TR36948-1, TR46861-3, TR44388-6, TR44433-5, TR48373-1, } \\
\text { TR54988-1, TR54989-4, TR37383 }\end{array}$ & 17 \\
\hline $\begin{array}{l}\text { Eastern } \\
\text { Anatolia }\end{array}$ & $\begin{array}{l}\text { TR32650-5, TR32668-2, TR32780-2, TR32846-6, TR45370-2, TR48034-6, TR32231-5, } \\
\text { TR32231-6, TR45420-5, TR45422-2, TR32273-4, TR39676-4, TR45402-3, TR45398-4, } \\
\text { TR47993-1, TR32761-6, TR32881-2, TR39660-1, TR45105-6, TR48025-3, TR47961, } \\
\text { TR48050, TR31894-3, TR31894-6, TR32014-5, TR32218, TR63329, TR63322 }\end{array}$ & 28 \\
\hline $\begin{array}{l}\text { Southeastern } \\
\text { Anatolia }\end{array}$ & $\begin{array}{l}\text { TR32218-1, TR32218-5, TR32218-6, TR50443, TR46810-5, TR46822-2, TR38888-6, } \\
\text { TR50455-4, TR49018-1, TR31678 }\end{array}$ & 10 \\
\hline
\end{tabular}


Table 2. The sequence data for the primers used and expected fragment sizes.

\begin{tabular}{|c|c|c|c|c|}
\hline & Alleles & Primer sequences & $\begin{array}{c}\text { Expected fragment } \\
\text { sizes (bp) } \\
\end{array}$ & References \\
\hline P1 & Dx2, Dx 5 & $\begin{array}{l}\text { F: GCCTAGCAACCTTCACAATC } \\
\text { R: GAAACCTGCTGCGGACAAG }\end{array}$ & 450 & Ahmad (2000) \\
\hline $\mathbf{P 2}$ & Dy10, Dy12 & $\begin{array}{l}\text { F: GTTGGCCGGTCGGCTGCCATG } \\
\text { R: TGGAGAAGTTGGATAGTACC }\end{array}$ & $\begin{array}{l}576 \\
612\end{array}$ & Ahmad (2000) \\
\hline $\mathbf{P 3}$ & $\begin{array}{l}\text { By } 18^{*}, \text { By } 20 *, \text { By } 8, \\
\text { By8*,By9,By16 }\end{array}$ & $\begin{array}{l}\text { F:GCAGTACCCAGCTTCTCAA } \\
\text { R:CCTTGTCTTGTTTGTTGCC }\end{array}$ & $290-400$ & Salmanowicz\&Dylewicz (2007) \\
\hline $\mathbf{P 4}$ & Axnull & $\begin{array}{l}\text { F: ACGTTCCCCTACAGGTACTA } \\
\text { R:TATCACTGGCTAGCCGACAA }\end{array}$ & 920 & Salmanowicz\&Dylewicz (2007) \\
\hline P5 & $A x 1+A x 2 *$ & $\begin{array}{l}\text { F:CCATCGAAATGGCTAAGCGG } \\
\text { R:GTCCAGAAGTTGGGAAGTGC }\end{array}$ & 1500 & Salmanowicz\&Dylewicz (2007) \\
\hline P6 & $A x 2 *$ & $\begin{array}{l}\text { F:CCGATTTTGTTCTTCTCACAC } \\
\text { R:CACCAAGCGAGCTGCAGAT }\end{array}$ & 2652 & Salmanowicz \& Dylewicz (2007) \\
\hline P7 & $\begin{array}{l}\text { By } 20^{*}, \text { By } 8, \text { By } 8 * \\
\text { By } 18^{*}, \text { By } 9\end{array}$ & $\begin{array}{l}\text { F:TTCTCTGCATCAGTCAGGA } \\
\text { R:AGAGAAGCTGTGTAATGCC }\end{array}$ & $750,710,660$ & Salmanowicz \& Dylewicz (2007) \\
\hline P8 & $B x 7$ & $\begin{array}{l}\text { F:ATGGCTAAGCGCCTGGTCCT } \\
\text { R:TGCCTGGTCGACAATGCGTCGCTG }\end{array}$ & 2373 & Ahmad (2000) \\
\hline P9 & By8 & $\begin{array}{l}\text { F:TTAGCGCTAAGTGCCGTCT } \\
\text { R:TTGTCCTATTTGCTGCCCTT }\end{array}$ & 527 & Salmanowicz \& Dylewicz (2007) \\
\hline P10 & $\begin{array}{l}B \times 14 \text { and } B \times 17 \\
\quad \text { cauBx752 }\end{array}$ & $\begin{array}{l}\text { F:AGGGGCAGGGAAGAAACACT } \\
\text { R:CCAGGCAACACAAATCCATG }\end{array}$ & 642 and 534 & Xu et al. (2008) \\
\hline P11 & $\begin{array}{l}B \times 14 \text { and } B \times 17 \\
\text { CauBx642 }\end{array}$ & $\begin{array}{l}\text { F:GGGCAATCGGGGTACTTCC } \\
\text { R:CCCTTGTCTTGGCTGTTGTC }\end{array}$ & 642 and 534 & Xu et al. (2008) \\
\hline P12 & $\mathrm{Ax} 2^{*}$ & $\begin{array}{l}\text { F: ATGACTAAGCGGTTGGTTCTT } \\
\text { R: ACCTTGCTCCCCTTGTCTTT }\end{array}$ & 1400 & Ma et al. (2003) \\
\hline P13 & $\mathrm{Bx}$ & $\begin{array}{l}\text { F: CGCAACAGCCAGGACAATT } \\
\text { R: AGAGTTCTATCACTGCCTGGT }\end{array}$ & $650-750$ & Ma et al. (2003) \\
\hline P14 & $\mathrm{Bx} 6, \mathrm{Bx} 7, \mathrm{Bx} 7 *$ & $\begin{array}{l}\text { F:CAAGGGCAACCAGGGTAC } \\
\text { R:AGAGTTCTATCACTGCCTGGT }\end{array}$ & $\begin{array}{l}(850-920),(420-640) \\
(180-280)\end{array}$ & Salmanowicz \& Dylewicz (2007) \\
\hline P15 & GluD1y10 & $\begin{array}{l}\text { F:5'-CAACCAATCTCCACAATC-3' } \\
\text { R:5'-CTGCAGAGAGTTCTATCA-3' }\end{array}$ & 2210 & De Bustos \& Jouve (2003) \\
\hline P16 & $\begin{array}{c}\text { GluA1-1, 2* } \\
\text { Co-dominant Ax2* }\end{array}$ & $\begin{array}{l}\text { F:5'-AAGACAAGGGGAGCAAGGT-3' } \\
\text { R:5'- GTGCTCCGCGCTAACATG-3' }\end{array}$ & $\begin{array}{l}1090 \\
1063\end{array}$ & Radovanovic \& Cloutier (2003) \\
\hline P17 & $\begin{array}{l}\text { GluB1 } 7^{*}, \mathrm{H} 7 \\
\text { Dominant Bx7 }\end{array}$ & $\begin{array}{l}\text { F:5'-CAACAACTTGTGGGGGCCTT-3' } \\
\text { R:5'-GCGCTTAGCCATCTCAGTGAAC-3' }\end{array}$ & 1116 & Radovanovic \& Cloutier (2003) \\
\hline P18 & Co-dominant Bx7 & $\begin{array}{l}\text { F:5'-ACCTCAGCATGCAAACATG-3' } \\
\text { R:5'- GCGCTTAGCCATCTCAGTGAAC-3' }\end{array}$ & $\begin{array}{l}530,1259,1302 \\
3200\end{array}$ & Radovanovic \& Cloutier (2003) \\
\hline P19 & $\begin{array}{l}\text { GluD1-2, } 5 \text { Dx } 5 \\
\text { Dominant }\end{array}$ & $\begin{array}{l}\text { F:5'-CGTCCCTATAAAAGCCTAGCC-3' } \\
\text { R:5'-GGCTAATGTCTCGGAGCTGT-3' }\end{array}$ & 272 & Radovanovic \& Cloutier (2003) \\
\hline $\mathbf{P 2 0}$ & $\begin{array}{c}\mathrm{AX} \\
\mathrm{Ax} 2^{*}, \mathrm{Ax} 1\end{array}$ & $\begin{array}{l}\text { F:5'-ATGACTAAGCGGTTGGTTCTT-3' } \\
\text { R:5'-GACCTTGCTCCCCTTGTCTTT-3' }\end{array}$ & 1319 & Ma et al. (2003) \\
\hline P21 & $\begin{array}{c}\mathrm{AX} \\
\mathrm{Ax} 2^{*}, \mathrm{Ax} 1\end{array}$ & $\begin{array}{l}\text { F:5'-ATGACTAAGCGGTTGGTTCTT-3' } \\
\text { R:5'-GACCTTGCTCCCCTTGTCCTG-3' }\end{array}$ & 1500 & Ma et al. (2003) \\
\hline $\mathbf{P 2 2}$ & Dy10 & $\begin{array}{l}\text { F:5'-GACAGTCCACCGAGATGG-3' } \\
\text { R:5'-GCAAGCTGCAGAGAGTTC-3' }\end{array}$ & 1400,2000 & Mishra et al. (2009) \\
\hline $\mathbf{P 2 3}$ & Dx5 & $\begin{array}{l}\text { F:5'-CATGGTCCTGAACCTTCACC-3' } \\
\text { R:5'-CAGAGAGTTCTATCACTGGC-3' }\end{array}$ & 2000 & Mishra et al. (2009) \\
\hline P24 & $1 \mathrm{Ax} 1(\mathrm{Ax} 2)$ & $\begin{array}{l}\text { F:5'-CCGAGATGACTAAGCGG-3' } \\
\text { R:5'-GCTAACATGGTATGGGCT-3' }\end{array}$ & 1800,2500 & Mishra et al. (2009) \\
\hline P25 & $\begin{array}{l}\text { Dx5 } \\
\text { Dx2 }\end{array}$ & $\begin{array}{l}\text { F:5'-CGTCCCTATAAAAGCCTAG-C-3' } \\
\text { R:5'-AGTATGAAACCTGCTGCGGAC-3' }\end{array}$ & 478 & Ma et al. (2003) \\
\hline P26 & $\begin{array}{l}\text { Dx5 } \\
\text { Dx2 }\end{array}$ & $\begin{array}{l}\text { F:5'-CGTCCCTATAAAAGCCTAG-C-3' } \\
\text { R:5'-AGTATGAAACCTGCTGCGGAG-3' }\end{array}$ & 450 & Ma et al. (2003) \\
\hline P27 & $\begin{array}{l}\text { Dx5 } \\
\text { Dx2 }\end{array}$ & $\begin{array}{l}\text { F:5'CGTCCCTATAAAAGCCTAGTT-3' } \\
\text { R:5'-AGTATGAAACCTGCTGCGGAC-3' }\end{array}$ & 450 & Ma et al. (2003) \\
\hline P28 & $\begin{array}{l}\text { Dx5 } \\
\text { Dx2 }\end{array}$ & $\begin{array}{l}\text { F:5'-CGTCCCTATAAAAGCCTAGTT-3' } \\
\text { R:5'-AGTATGAAACCTGCTGCGGAG-3' }\end{array}$ & 450 & Ma et al. (2003) \\
\hline P29 & 1Dy10.1 & $\begin{array}{l}\text { F:5'-ATGGCTAAGCGGC/TTA/GGTCCTCTTTG-3' } \\
\text { R:5'-CTATCACTGGCTG/AGCCGACAATGCG-3' }\end{array}$ & 372 & Jiang et al. (2006) \\
\hline P30 & degenere primer & $\begin{array}{l}\text { F:ATCACCCACAACACCGAGCA-3’G } \\
\text { R:CTATCACTGGCTA/GGCCGACAATGCG }\end{array}$ & 1800,2000 & Ma et al. (2003) \\
\hline P31 & degenere primer & $\begin{array}{l}\text { F:AGGGAAAGACAATGGACATG } \\
\text { R:TAGTTG/TCCC/TAGAGGCCTCACCTTC } \\
\end{array}$ & $\begin{array}{c}1800,2000,2100, \\
2500 \\
\end{array}$ & Jiang et al. (2006) \\
\hline
\end{tabular}




\section{Materials and Methods}

\section{Plant materials}

116 bread wheat landraces of Turkey obtained from Ankara Field Crops Central Research Institute and Izmir Aegean Agricultural Research Institute were included in the study (Table 1). The specimens were so selected to provide representation of all geographical regions (Aegean, Central Anatolia, Marmara, the Mediterranean, Black Sea, Eastern Anatolia and Southeastern Anatolia) in the country. These were supplied from the "Field Crops Central Research Institute, Ankara" and "Aegean Agricultural Research Institute, İzmir. 15 standard genotypes (Chinese Spring, Drago, Lobeiro, Svevo, Lira, Durambo, Ak 702, Bezostaya 1, KateA1, Bayraktar, Mizrak, Yakar, Atay 85, Gerek 79 and Tosunbey) were used as references to define gluten alleles The wheat specimens studied with their respective distribution regions is provided in Table 1 .

\section{DNA extraction and PCR analysis}

DNA was isolated from the seeds according to the method described by McCarthy et al. (2002) using 31 primer pairs (Table 2). PCR reaction was realized in $15 \mu \mathrm{l}$ reaction mixture containing $50 \mathrm{nmol}$ of each primer, 0.3 nmol dNTP, 1-2 unit Taq polymerase, 1.5-2.5 $\mathrm{mM} \mathrm{MgCl}_{2}$, $10 \mu \mathrm{g} / \mu \mathrm{l} \mathrm{BSA}$ and $30 \mathrm{ng}$ template DNA. Following the initial denaturation at $94^{\circ} \mathrm{C}$ for 5 minutes, PCR reaction was carried out in 35 cycles of $94^{\circ} \mathrm{C}$ for 1 minute, $54-65^{\circ} \mathrm{C}$ for $1-2$ minutes and $72^{\circ} \mathrm{C}$ for 2 minutes. The last extension step was realized at $72^{\circ} \mathrm{C}$ for 10 minutes. PCR products were run in $1-1.5 \%$ agarose-containing ethidium bromide and imaged with a gel documentation system (BioRad MP5 gel documentation system).

\section{Gluten extraction and SDS-PAGE analyses}

Gluten proteins were extracted in accordance with the methods described by Gao et al. (2012) and Temizgul et al. (2018). The methods of Li et al. (2012) with slight modification were used for the electrophoresis of gluten proteins. 30-60 mA current was applied per gel during electrophoresis. Gels were stained (dissolved in $187.5 \mathrm{ml}$ of methanol, $225 \mathrm{mg}$ of CBB-R 250; $750 \mathrm{ml} \mathrm{10 \%} \mathrm{TCA}$ and $62.5 \mathrm{ml}$ glacial acetic acid) for 12-24 hours and kept in washing solution (333 ml methanol, $100 \mathrm{ml} \mathrm{10 \%} \mathrm{TCA}$ and $567 \mathrm{ml}$ distilled water) for 5 hours and imaged under the gel documentation system.

\section{Definition of gluten alleles}

Gluten alleles of the wheat landraces were defined based on individual HMW subunit distributions in SDSPAGE in accordance with the methods specified by Payne \& Lawrence (1983) and McIntosh et al. (1994). The verification of alleles defined by allele-specific primers was also performed.

\section{Calculation of Glu-1 quality scores}

Based on the SDS-PAGE profile, relationships between individual HMW subunits and quality was determined by using the method described by Payne (1987a) and scored by using subunit scores of Payne (1987a) and Lukow et al. (1989).

POPGENE version 1.31 software was used to draw dendrogram based on Nei's original measurement showing the relationships among the genotypes based on individual subunits (Nei 1972).

\section{Statistical analysis}

Data analysis was carried out based on the frequencies of HMW gluten gene alleles. Statistical analyses were performed separately in individual variety and populations for individual alleles, individual sub-units, loci (Glu-Al, Glu-B1, and Glu-Dl) and geographical origin (7 regions). POPGENE version 1.31 software was used for statistical analysis. Polymorphism percentages were calculated using Equation 1;

Eq. 1: Polymorphism\% = (number of polymorphic allele/number of total alleles $) \times 100$.

Allele frequency of $G l u-1$ loci $(G l u-A 1, G l u-B 1$, and Glu-D1) was calculated according to Gupta et al. (1991). Percentage allele frequencies were calculated by using Equation 2;

Eq. 2: Allele frequency $=$ (number of individual alleles/number of total wheat samples $) \times 100$.

Allelic combination frequencies were calculated using Equation 3;

Eq. 3: Allelic combination frequency $=($ observed total number of each allelic combinations/number of total wheat samples) $\times 100$.

\section{Results}

In A genome of studied genotypes, the frequencies of Glu-Alc, Glu-Alb, and Glu-Ala were calculated to be $65.1 \%, 26.4 \%$, and $8.5 \%$, respectively (Table 3). In B genome, the frequencies of Glu-Blb, Glu-Ble, Glu-Bld, and $G l u-B l c$ were determined to be $44.6 \%, 16.6 \%$, $10.0 \%$, and $10.7 \%$, respectively. In $\mathrm{D}$ genome, the frequencies of Glu-Dla, Glu-Dld, and Glu-Dlb were determined to be $59.7 \%, 27.9 \%$, and $7.7 \%$, respectively. Since quality scores were not determined for these subunits, the contributions of the alleles Glu-Blh, Glu$B l z$, and $G l u-B l$ aj to quality score could not be calculated for 7 of the 116 genotypes studied. After determination of rheological characteristics of individual subunits, the contribution of these subunits to the quality can also be determined. The average quality score in the genotypes was calculated to be 6.07. The Marmara and the Southeastern Anatolia regions had 7.18 and 4.8 quality scores as the highest and the lowest, respectively.

\section{Subunit frequencies}

Total and region-based subunit frequencies were given in Table 3. The highest frequencies were observed as $65.11 \%, 54.30 \%$, and $58.30 \%$ for the subunits Glu-Alc, Glu-Blb, and Glu-Dla, respectively. Glu-l genome subunit frequencies are given in Table 3 . Based on the 
regions, the frequencies of Glu-Ala (18.2\%), Glu-Alb (50.0\%), Glu-Alc (80.0\%), Glu-Blb (58.0\%), Glu-Ble (46.0\%), Glu-Dla (86.7\%), Glu-Dld (50.0\%), were the highest in Mediterranean, Marmara, Aegean, Black Sea, Southeastern Anatolia, Aegean and Marmara, respectively.

Table 3. Total and region-based subunit frequencies (\%).

\begin{tabular}{|c|c|c|c|c|c|c|c|c|c|}
\hline Sub & Regions & 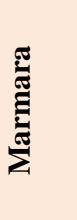 & 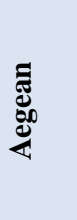 & 胥 & 丞 & 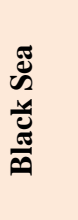 & 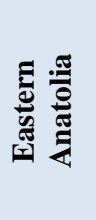 & 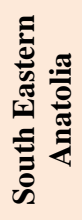 & 롤 \\
\hline \multicolumn{10}{|c|}{ Glu-AI } \\
\hline a & 1 & 14.2 & 13.3 & 18.2 & - & 15.8 & 3.3 & 8.3 & 8.5 \\
\hline$b$ & $2 *$ & 50.0 & 6.7 & 18.2 & 32.1 & 31.6 & 23.3 & 16.7 & 26.4 \\
\hline $\mathrm{c}$ & Null & 35.8 & 80.0 & 63.6 & 67.9 & 52.6 & 73.3 & 75.0 & 65.1 \\
\hline \multicolumn{10}{|c|}{ Glu-B1 } \\
\hline $\mathrm{a}$ & 7 & 7.0 & 5.0 & - & - & - & - & - & 1.7 \\
\hline$b$ & $7+8$ & 27.5 & 56.0 & 17.0 & 52.0 & 58.0 & 56.0 & 46.0 & 44.6 \\
\hline $\mathrm{c}$ & $7+9$ & 32.0 & - & 8.0 & 14.0 & 9.0 & 3.3 & 8.0 & 10.7 \\
\hline d & $6+8$ & - & 24.0 & 8.0 & 31.0 & 4.0 & 3.0 & - & 10.0 \\
\hline e & 20 & 13.0 & 5.0 & 17.0 & 3.0 & 20.0 & 12.3 & 46.0 & 16.6 \\
\hline f & $13+16$ & - & - & - & - & - & 6.0 & - & 0.9 \\
\hline $\mathrm{h}$ & $14+15$ & - & - & 50.0 & - & - & 3.3 & - & 7.6 \\
\hline I & $17+18$ & - & 5.0 & - & - & - & - & - & 0.7 \\
\hline $\mathrm{u}$ & $7 *+8$ & - & - & - & & - & 6.7 & - & 1.0 \\
\hline aj & 8 & 7.0 & 5.0 & - & & 9.0 & - & - & 3.0 \\
\hline $\mathrm{z}$ & $7+15$ & 13.5 & - & - & & - & 9.4 & - & 3.2 \\
\hline \multicolumn{10}{|c|}{ Glu-D1 } \\
\hline $\mathrm{a}$ & $2+12$ & 50.0 & 86.7 & 45.5 & 67.9 & 63.2 & 40.0 & 58.3 & 58.3 \\
\hline$b$ & $3+12$ & - & 6.7 & 22.0 & 10.7 & - & 10.0 & 8.3 & 8.2 \\
\hline $\mathrm{c}$ & $4+12$ & - & - & 10.5 & 3.6 & 5.3 & 3.3 & 8.3 & 4.3 \\
\hline d & $5+10$ & 50.0 & 6.7 & 22.0 & 17.9 & 31.6 & 40.0 & 25.0 & 27.6 \\
\hline $\mathrm{h}$ & $2+12 *$ & - & - & - & - & - & 6.7 & - & 1.6 \\
\hline
\end{tabular}

Table 4. Allelic combinations and the frequencies.

\begin{tabular}{|c|c|c|c|c|c|}
\hline Allelic combinations & $\begin{array}{c}\text { Subunit } \\
\text { combinations }\end{array}$ & $\begin{array}{c}\text { Frequency } \\
(\%)\end{array}$ & Allelic combinations & $\begin{array}{c}\text { Subunit } \\
\text { combinations }\end{array}$ & $\begin{array}{c}\text { Frequency } \\
(\%)\end{array}$ \\
\hline Glu-Blb/Glu-Dla & $(7+8,2+12)$ & 22.48 & Glu-Alb/Glu-Bld/Glu-Dlc & $(2 *, 6+8,4+12)$ & 0.78 \\
\hline Glu-Alc/Glu-Blb/Glu-Dla & $($ Null, $7+8,2+12)$ & 11.70 & $G l u-A 1 \mathrm{a} / G l u-B 1 \mathrm{c} / G l u-D 1 \mathrm{a}$ & $(1,7+9,2+12)$ & 0.78 \\
\hline Glu-Alb/Glu-B1c/Glu-DId & $(2 *, 7+9,5+10)$ & 9.35 & $G l u-A 1 \mathrm{~b} / G l u-B 1 \mathrm{c} / G l u-D 1 \mathrm{~b}$ & $(2 *, 7+9,3+12)$ & 0.78 \\
\hline$G l u-B 1 \mathrm{~d} / G l u-D 1 \mathrm{a}$ & $(6+8,2+12)$ & 5.45 & $G l u-B 1 \mathrm{z} / G l u-D 1 \mathrm{~d}$ & $(7+15,5+10)$ & 0.78 \\
\hline Glu-AIb/Glu-Blb/Glu-DId & $(2 *, 7+8,5+10)$ & 4.70 & $G l u-B l \mathrm{e} / G l u-D l \mathrm{~d}$ & $(20,5+10)$ & 0.78 \\
\hline$G l u-B 1 \mathrm{~b} / G l u-D 1 \mathrm{~d}$ & $(7+8,5+10)$ & 4.70 & $G l u-A 1 \mathrm{c} / G l u-B 1 \mathrm{~b} / G l u-D 1 \mathrm{~d}$ & (Null, $7+8,5+10)$ & 0.78 \\
\hline Glu-Alc/Glu-Bld/Glu-Dla & (Null, 6+8, 2+12) & 3.15 & $G l u-B 1 \mathrm{e} / G l u-D l \mathrm{c}$ & $(20,4+12)$ & 0.78 \\
\hline Glu-Ble/Glu-Dla & $(20,2+12)$ & 3.15 & Glu-Blb/Glu-Dlc & $(7+8,4+12)$ & 0.78 \\
\hline$G l u-A 1 \mathrm{~b} / G l u-B 1 \mathrm{~d} / G l u-D 1 \mathrm{~b}$ & $(2 *, 6+8,3+12)$ & 2.35 & Glu-Alb/Glu-Ble/Glu-Dld & $(2 *, 20,5+10)$ & 0.78 \\
\hline Glu-Ala/Glu-Ble/Glu-Dla & $(1,20,2+12)$ & 2.35 & Glu-Alb/Glu-Bli/Glu-Dld & $(2 *, 17+18,5+10)$ & 0.78 \\
\hline Glu-Alb-c/Glu-Blb/Glu-DIb & $(2 *, 7+8,3+12)$ & 1.56 & $G l u-A 1 \mathrm{a} / G l u-B 1 \mathrm{aj} / G l u-D 1 \mathrm{a}$ & $(1,8,2+12)$ & 0.78 \\
\hline Glu-A1c/Glu-B1z/Glu-D1a & (Null, $7+15,2+12)$ & 1,56 & Glu-Ala/Glu-Blf/Glu-Dla & $(1,13+16,2+12)$ & 0.78 \\
\hline$G l u-B 1 z / G l u-D 1 \mathrm{a}$ & $(7+15,2+12)$ & 1.56 & $G l u-A l \mathrm{a} / G l u-B l$ aj-e/Glu-Dld & $(1,8,20,5+10)$ & 0.78 \\
\hline$G l u-B 1 \mathrm{~b} / G l u-D 1 \mathrm{~b}$ & $(7+8,3+12)$ & 1.56 & $G l u-A 1 \mathrm{a} / G l u-B 1 \mathrm{e} / G l u-D 1 \mathrm{a}$ & $(1,20,2+12)$ & 0.78 \\
\hline$G l u-B 1 \mathrm{u} / G l u-D 1 \mathrm{a}$ & $(7 *+8,2+12)$ & 1.56 & $G l u-A 1 \mathrm{a} / G l u-B 1$ z/Glu-Dla & $(1,7+15,2+12)$ & 0.78 \\
\hline Glu-Alc/Glu-Ble/Glu-Dld & (Null, 20, 5+10) & 1.56 & Glu-Alb/Glu-Bld $/ G l u-D l \mathrm{~d}$ & $(2 *, 6+8,5+10)$ & 0.78 \\
\hline Glu-Alc/Glu-Ble/Glu-Dla & (Null, 20, 2+12) & 1.56 & Glu-Alb/Glu-BIh/Glu-Dld & $\left(2^{*}, 14+15,5+10\right)$ & 0.78 \\
\hline Glu-Alb/Glu-Blb/Glu-Dlc & $(2 *, 7+8,4+12)$ & 1.56 & $G l u-A 1 \mathrm{~b} / G l u-B 1 \mathrm{f} / G l u-D 1 \mathrm{~d}$ & $(2 *, 13+16,5+10)$ & 0.78 \\
\hline Glu-Alc/Glu-BIb/Glu-DIb & (Null, $7+8,3+12)$ & 1.56 & $G l u-A 1 \mathrm{a} / G l u-B l \mathrm{f}$ & $(1,13+16)$ & 0.78 \\
\hline Glu-Alb/Glu-Bla/Glu-Dld & $(2 *, 7,5+10)$ & 0.78 & & & \\
\hline
\end{tabular}


Table 5. Region-based allelic combinations and their frequencies.

\begin{tabular}{|c|c|c|c|c|c|}
\hline Region & Allelic combinations & Subunit combinations & $\begin{array}{c}\text { Frequency } \\
(\%)\end{array}$ & Glu-1 Score & $\begin{array}{c}\text { Average Glu-1 } \\
\text { score } \\
\end{array}$ \\
\hline \multirow{7}{*}{ 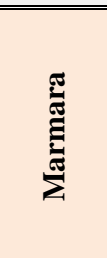 } & "Glu-Alb/Glu-Blc/Glu-Dld & $\left(2^{2 *}, 7+9,5+10\right)$ & 35.71 & 9 & \multirow{7}{*}{$7.18 \pm 2.34$} \\
\hline & Glu-Blb/Glu-Dla & $(7+8,2+12)$ & 14.29 & 5 & \\
\hline & Glu-Alb/Glu-Bla/Glu-Dld & $(2 *, 7,5+10)$ & 7.14 & 9 & \\
\hline & Glu-Ble/Glu-Dla & $(20,2+12)$ & 7.14 & 3 & \\
\hline & Glu-Ala/Glu-Ble/Glu-Dla & $(1,20,2+12)$ & 7.14 & 6 & \\
\hline & Glu-Alc/Glu-Blb/Glu-Dla & (null, $7+8,2+12$ ) & 7.14 & 6 & \\
\hline & $G l u-B 1 \mathrm{z} / G l u-D 1 \mathrm{a}$ & $(7+15,2+12)$ & 7.14 & $?$ & \\
\hline \multirow{5}{*}{ 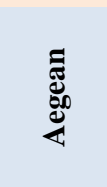 } & Glu-Alc/Glu-Blb/Glu-Dla & (null, $7+8,2+12$ ) & 26.67 & 6 & \multirow{5}{*}{$5.10 \pm 1.42$} \\
\hline & Glu-Blb/Glu-Dla & $(7+8,2+12)$ & 20.00 & 5 & \\
\hline & Glu-Alc/Glu-Bld/Glu-Dla & (null, $6+8,2+12)$ & 13.33 & 3 & \\
\hline & Glu-Alc/Glu-Blb/Glu-Dlb & (null, $7+8,3+12$ ) & 6.67 & 6 & \\
\hline & Glu-Ala/Glu-Blaj/Glu-Dla & $(1,8,2+12)$ & 6.67 & $?$ & \\
\hline \multirow{7}{*}{ } & Glu-Blb/Glu-Dla & $(7+8,2+12)$ & 18.18 & 5 & \multirow{7}{*}{$5.70 \pm 2.14$} \\
\hline & Glu-Blb/Glu-Dlb & $(7+8,3+12)$ & 18.18 & 5 & \\
\hline & Glu-Alb/Glu-Bld $/ G l u-D l \mathrm{~d}$ & $\left(2^{*}, 6+8,5+10\right)$ & 9.09 & 8 & \\
\hline & Glu-Alb/Glu-Blc/Glu-Dld & $(2 *, 7+9,5+10)$ & 9.09 & 9 & \\
\hline & Glu-Blb/Glu-Dlc & $(7+8,4+12)$ & 9.09 & 4 & \\
\hline & Glu-Ble/Glu-Dla & $(20,2+12)$ & 9.09 & 3 & \\
\hline & Glu-Alc/Glu-Blb/Glu-Dla & (null, $7+8,2+12$ ) & 9.09 & 6 & \\
\hline \multirow{8}{*}{ 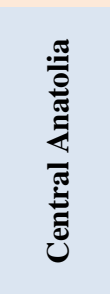 } & Glu-Blb/Glu-Dla & $(7+8,2+12)$ & 39.29 & 5 & \multirow{8}{*}{$5.63 \pm 2.38$} \\
\hline & $G l u-B l \mathrm{~d} / G l u-D l \mathrm{a}$ & $(6+8,2+12)$ & 14.29 & 3 & \\
\hline & Glu-Alb/Glu-Blc/Glu-Dld & $(2 *, 7+9,5+10)$ & 14.29 & 9 & \\
\hline & Glu-Alb/Glu-Blb/Glu-Dlb & $\left(2^{*}, 7+8,3+12\right)$ & 10.71 & 6 & \\
\hline & Glu-A1c/Glu-B1d/Glu-D1a & (null, $6+8,2+12$ ) & 7.14 & 4 & \\
\hline & Glu-Alb/Glu-Blb/Glu-Dld & $(2 *, 7+8,5+10)$ & 3.57 & 10 & \\
\hline & Glu-Alb/Glu-Blb/Glu-Dlc & $(2 *, 7+8,4+12)$ & 3.57 & 7 & \\
\hline & Glu-Alc/Glu-Blb/Glu-Dla & (null, $7+8,2+12$ ) & 3.57 & 6 & \\
\hline \multirow{10}{*}{ 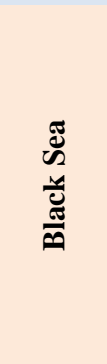 } & $G l u-B 1 \mathrm{~b} / G l u-D 1 \mathrm{a}$ & $(7+8,2+12)$ & 26.32 & 5 & \multirow{10}{*}{$6.65 \pm 1.73$} \\
\hline & Glu-Alc/Glu-Blb/Glu-Dla & (null, $7+8,2+12$ ) & 21.05 & 6 & \\
\hline & Glu-Alb/Glu-Blc/Glu-Dld & $(2 *, 7+9,5+10)$ & 5.26 & 9 & \\
\hline & Glu-Blb/Glu-Dld & $(7+8,5+10)$ & 5.26 & 7 & \\
\hline & Glu-Alb/Glu-Blb/Glu-Dld & $(2 *, 7+8,5+10)$ & 5.26 & 10 & \\
\hline & Glu-Alb/Glu-Bld/Glu-Dlc & $(2 *, 6+8,4+12)$ & 5.26 & 5 & \\
\hline & Glu-Alc/Glu-Blaj-e/Glu-Dla & (null, $8,20,2+12$ ) & 5.26 & $?$ & \\
\hline & Glu-Ala/Glu-Blc/Glu-Dla & $(1,7+9,2+12)$ & 5.26 & 7 & \\
\hline & Glu-Alb/Glu-Ble/Glu-Dld & $(2 *, 20,5+10)$ & 5.26 & 8 & \\
\hline & Glu-Ala/Glu-Ble/Glu-Dla & $(1,20,2+12)$ & 5.26 & 6 & \\
\hline \multirow{17}{*}{ 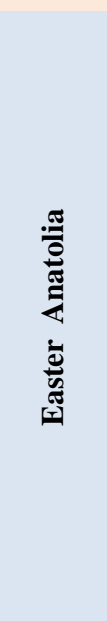 } & Glu-Blb/Glu-Dld & $(7+8,5+10)$ & 16.67 & 7 & \multirow{17}{*}{$6.57 \pm 2.10$} \\
\hline & Glu-Blb/Glu-Dla & $(7+8,2+12)$ & 10.00 & 5 & \\
\hline & Glu-Blu/Glu-Dla & $(7 *+8,2+12)$ & 6.67 & 5 & \\
\hline & Glu-Alc/Glu-Ble/Glu-Dla & (null, 20, 2+12) & 6.67 & 4 & \\
\hline & $G l u-A l \mathrm{c} / G l u-B l \mathrm{~h} / G l u-D 1 \mathrm{a}$ & (null, $14+15,2+12$ ) & 3.33 & $?$ & \\
\hline & Glu-Alb/Glu-Blb/Glu-Dld & $(2 *, 7+8,5+10)$ & 3.33 & 10 & \\
\hline & Glu-Alb/Glu-Blb/Glu-Dlb & $(2 *, 7+8,3+12)$ & 3.33 & 7 & \\
\hline & Glu-Alc/Glu-Bld/Glu-Dla & (null, $6+8,2+12$ ) & 3.33 & 4 & \\
\hline & Glu-Alc/Glu-Blz/Glu-Dla & (null, $7+15,2+12$ ) & 3.33 & $?$ & \\
\hline & $G l u-B 1 \mathrm{z} / G l u-D 1 \mathrm{~h}$ & $(7+15,2+12 *)$ & 3.33 & $?$ & \\
\hline & Glu-B1z/Glu-D1d & (null, $7+15,5+10)$ & 3.33 & $?$ & \\
\hline & $G l u-B l \mathrm{~b} / G l u-D l \mathrm{~h}$ & $(7+8,2+12 *)$ & 3.33 & 5 & \\
\hline & Glu-Alb/Glu-Blc/Glu-Dld & $(2 *, 7+9,5+10)$ & 3.33 & 9 & \\
\hline & Glu-Alc/Glu-Blb/Glu-Dld & (null, $7+8,5+10$ ) & 3.33 & 8 & \\
\hline & Glu-Alb-c/Glu-Blf/Glu-Dld & $(2 *, 13+16,5+10)$ & 3.33 & 10 & \\
\hline & Glu-Alc/Glu-Blb/Glu-Dlb & (null, $7+8,3+12$ ) & 3.33 & 6 & \\
\hline & Glu-Alc/Glu-Ble/Glu-Dld & (null, 20, 5+10) & 3.33 & 6 & \\
\hline \multirow{7}{*}{ 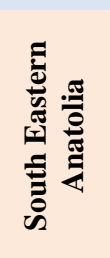 } & Glu-Blb/Glu-Dla & $(7+8,2+12)$ & 16.67 & 5 & \multirow{7}{*}{$4.80 \pm 1.77$} \\
\hline & Glu-Ble/Glu-Dla & $(20,2+12)$ & 16.67 & 3 & \\
\hline & Glu-Alc/Glu-Blb/Glu-Dla & (null, $7+8,2+12$ ) & 16.67 & 6 & \\
\hline & Glu-Ble/Glu-Dlc & $(20,4+12)$ & 8.33 & 2 & \\
\hline & Glu-Alb/Glu-Blc/Glu-Dlb & $(2 *, 7+9,3+12)$ & 8.33 & 7 & \\
\hline & Glu-Alc/Glu-Ble/Glu-Dld & (null, 20,5+10) & 8.33 & 6 & \\
\hline & Glu-Ble/Glu-Dld & $(20,5+10)$ & 8.33 & 5 & \\
\hline
\end{tabular}




\section{Allelic combinations and frequencies}

Allelic combinations and their frequencies are given in Table 4. The results indicated the presence of 39 different allelic combinations. Among these combinations, Glu-Blb/Glu-Dla was the most frequent combination with a $22.48 \%$ frequency value, followed by Glu-Alc/Glu-Blb/Glu-Dla, Glu-Alb/Glu-Blc/Glu-Dld, and $G l u-B l \mathrm{~d} / G l u-D 1 \mathrm{a}$ allelic combinations with $11.7 \%$, $9.35 \%$, and $5.45 \%$ frequencies, respectively. The rest of the alleles were observed with a frequency less than $5 \%$.

\section{$\underline{\text { Region-based allelic combinations and Glu-1 scores }}$}

The frequencies of HMW gluten subunits and their Glu-1 scores are given in Table 5. In some of the genotypes, the effect of some subunits on quality score could not be calculated since the effect of these subunits on quality score was not determined. The quality score was 10 in four accessions (TR32846-6, TR36948-1, TR45105 and TR63536), and 9 in 6 accessions (TR45398-4, TR48025-3, TR33264-6, 393-5, TR520213 , TR45094). The highest (17) and the lowest (5) allelic combinations were observed in Eastern Anatolia and Aegean regions, respectively (Table 5).

\section{Development of dendrogram based on subunits of Glu-1} $\underline{\text { loci }}$

When the dendrograms (Nei 1972) drawn based on individual subunits were investigated, the Mediterranean region clustered with Black sea (genetic distance 0.32) and Aegean region clustered with Central Anatolia (genetic distance 0.52) (Fig. 1). Marmara (genetic distance 1.35), Eastern Anatolia (genetic distance 0.37), and Southeastern Anatolia (genetic distance 0.52) regions were separately clustered.

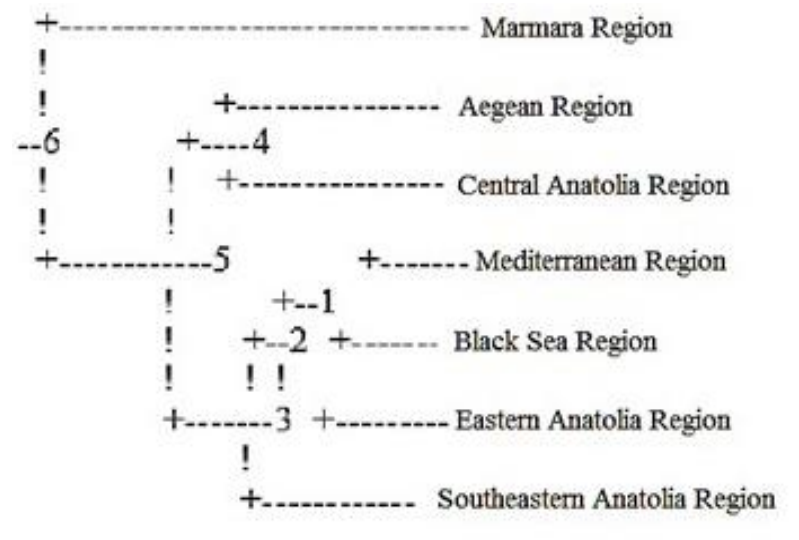

\begin{tabular}{lllllll}
\hline 1.35 & 0.65 & 0.52 & 0.37 & 0.32 & 0.00
\end{tabular}

Fig. 1. Dendrogram showing the relationships of the genotypes based on individual subunits of Glu-l loci.

\section{Discussion}

The determination of HMW-GS composition in wheat cultivar collections from different countries has been studied (Nucia et al. 2019). The contribution of individual subunits on dough quality is quite different (Payne et al. 1987, Lukow et al. 1989). While quality score of Glu-Dld is the highest (quality score 4), the contribution of GluAlc, Glu-Bla, Glu-Bld, Glu-Ble, and Glu-Dlc is considered to be the lowest (quality score 1). In the present study, the highest Glu-Dld frequency $(50 \%)$ was observed in Marmara and the lowest was observed in Aegean regions (6.67\%). The frequency of this subunit was $27.6 \%$ throughout Turkey (Table 2). The highest Glu1 score with 7.18 was observed in Marmara followed by Black Sea with 6.65, Eastern Anatolia with 6.57, Mediterranean with 5.70, Central Anatolia with 5.63, Aegean with 5.10, and Southeastern Anatolia regions with 4.8 (Table 5).

Observing the highest quality score in Marmara and the lowest in the Southeastern region is an expected outcome. Southeastern Turkey is on the interception of gene centers for wheat and its wheat is intensively studied and high-quality score wheat genotypes are already selected for breeding. Marmara region is the most industrialized region and agricultural activity is quite poor in this region, therefore, the landraces might not be used to select for high-quality score cultivar breeding. Throughout Turkey, 19 different subunits (3 Glu-Al, 11 Glu-Bl and 5 Glu-Dl) were observed in 39 different combinations (Tables 3 and 4). Among the allelic combinations $G l u-B l \mathrm{~b} / G l u-D l$ a was the highest with $22.48 \%$ frequency, followed by Glu-Alc/Glu-Blb/Glu$D l$ a with $11.70 \%$, Glu-Alb/Glu-Blc/Glu-Dld with $9.35 \%$ and $G l u-B l d / G l u-D l$ a with $5.45 \%$. Other alleles were observed with less than $5 \%$ frequency. The highest allelic variation with 16 subunits and 17 different combinations was observed in Southeastern Anatolia. Glu-Blb/Glu-Dld allelic combinations with $16.67 \%$ frequency were the most frequent allelic combinations in this region (Table 5). Based on Nei's original measurement (Nei 1972), while the highest similarity among regions for individual subunits of Glu-1 loci was observed between Black Sea and the Mediterranean regions (0.9935), the lowest similarity was observed in Marmara and the Aegean regions (0.953). When the dendrogram was drawn based on Nei's (1972) original measurements, the Mediterranean clustered with the Black Sea and the Aegean with the Central Anatolia accessions. Other regions were separately clustered (Fig. 1).

Nakamura et al. (1999) studied variations in the HMW subunits of Glu-1 loci of Kapon wheats and identified 14 different alleles, 3 of which were on Glu-A1, 6 on Glu-B1 and 5 on $G l u-D 1$ loci. In the present study, 19 different subunits were identified and 3 of them were on $G l u-A l$, 11 of them on Glu-Bl and 5 of them on Glu-Dl loci. The frequency of null alleles located on chromosome $1 \mathrm{~A}$ was reported to be high (74\%) on Japanese wheat especially on Norin variety. The frequency of this allele was also found to be high in landraces of Turkish wheat $(65.11 \%)$. While Glu-Bla, Glu-Blf, Glu-Blh, Glu-Blj, and Glu-Blk subunits were not observed in Japanese bread wheat (Nakamura et al. 1999), only Glu-Blj and Glu-Blk 
subunits were not observed in Turkish wheat genotypes used in this study. While the frequency of $2+12$ subunit coded by Glu-Dla allele was $55 \%$ in Japanese wheat, it was only $1.5 \%$ in $5+10$ subunit coded by Glu-Dld. The average frequencies of these alleles were found to be $58.3 \%$ and $27.6 \%$ in Turkish genotypes, respectively. The frequencies of Glu-Dla and Glu-Dld subunits were $86.7 \%$ and $50 \%$ in Aegean and Marmara regions, respectively. The frequency of Glu-Dld subunit was found to be high in European wheat varieties (Payne et al. 1984). The frequency of this subunit was also found to be high in Turkish wheat landraces, especially in Marmara region (average 27.6\%, Marmara region 50.0\%). Cabellero et al. (2009) investigated the seed storage protein diversity of the wild diploid wheat genotypes obtained from Lebanon and Turkey. They determined 10 alleles at Glu-A1, 16 alleles at Glu-A3, 15 alleles at Gli$A 1,18$ alleles at Gli-A2 and detected 4 frequent, 2 infrequent, and 3 rare alleles. In the present study, 11 frequent, 7 infrequent and 4 rare alleles were determined.

Van Hintum \& Elings (1991) evaluated the Syrian durum wheat genotypes based on phenotype and gluten content. They observed 19 HMW subunits in 48 different combinations. In the present study, 22 individual alleles and 19 HMW subunits were detected in 39 different combinations. Gianibelli et al. (2002) studied molecular and biochemical characterizations of Argentinian wheat cultivars, identified the allelic variations, and calculated the allele frequencies. Of the 11 alleles, 3 were coded by $G l u-A 1,6$ were coded by $G l u-B 1$ and 2 were coded by $G l u-D 1$ loci. Null allele frequency was found to be quite low $(1.1 \%)$ and $G l u-D l d$ was the highest in frequency. However, Glu-Alc subunit was the highest with the frequency of $65.1 \%$ among the alleles in Turkish landraces. Gianibelli et al. (2002) also calculated the quality score taking the $G l u-A l$ into account and separated the Argentinian wheat into 18 groups. Glu-Ala/Glu$B l \mathrm{bq} / G l u-D l d$ was observed in highest frequency (22\%). In Turkish landraces, Glu-Blb/Glu-Dla was observed in highest frequency.

Payne (1987b) found the quality score of world wheat collection as 9.5 . The quality score of Turkish wheat landraces was found to be significantly lower (6.07). Notwithstanding, quality score is not determined by only HMW-GS, the contribution of LMW-GS and Gliadins should also be taken into account (MacRitchie et al. 1990).

Payne \& Lawrence (1983) published the catalogue of Glu- 1 alleles. They determined 3, 11, and 7 alleles in Glu$A 1, G l u-B 1$, and $G l u-D 1$, respectively. Additional alleles were also determined but most of those alleles were found to be in Glu-Bl loci (Pogna et al. 1990). In the present study, a possible new allele (Dy12*) was determined in Glu-D1 locus. This new subunit was differentiated considering its faster movement in SDS-PAGE.

Branlard et al. (1989) observed 3 allelic variations in Glu-Al of 165 Turkish durum wheat cultivars. Glu-Al-1 allele was coding an $\mathrm{x}$ subunit that had bigger electrophoretic mobility than $2 *$. The researchers suggested that this allele was similar to previously discovered two alleles (Glu-AlV, Glu-AlVI). The null allele with $68.9 \%$ frequency was the most frequent allele followed by Glu-Alb (28.3\%) and Glu-Al-1 (3.8\%). Seven different $G l u-B 1$ allele variants were identified in previous studies subjecting the Turkish wheats (Branlard et al. 1989). These consisted of 5 different $x$ and y type subunit combinations. Of the $7 G l u-B 1,5$ (Glu-Blb, Glu$B 1 \mathrm{~d}, G l u-B l \mathrm{e}, G l u-B l \mathrm{~h}$, and $G l u-B 1 \mathrm{z})$ were observed among Turkish wheat samples (Branlard et al. 1989, Payne et al. 1981). In the present study, 11 subunits were determined in Glu-B1 loci (Table 3).

Primitive cultivars and locally grown landraces were considered to be the sources of variation for grain protein quality, disease resistance, and resistance to abiotic stress conditions (Porceddu et al. 1988, Kaplan et al. 2014). HMW subunit variations in Turkish bread wheat landraces were found to be higher compared to Australian, Italian, American, Canadian, French and Spanish wheat samples (Autran \& Feillet 1985, Margiotta et al. 1987, Carrillo et al. 1990). This outcome is somewhat expected since intensive breeding works have decreased the variation in western wheat genotypes (Porceddu et al. 1988).

Morgunov et al. (1993) and Sultana et al. (2007) stated that the increase in HMW score was related to a decrease in diversity in gluten alleles. A similar phenomenon was also observed in wheats grown in Dobrudzha Agricultural Institute (Atanasova et al. 2009). That is why it is crucial to use Glu-Blf, Glu-Blh and Glu-Bli subunits to increase quality score. This situation may decrease genetic diversity and increase end-product quality (Liu et al. 2007). In the present study, the observation of these alleles was also quite low in 116 Turkish wheat landraces (approximately 7\%). The frequency of Glu-Blh (14+15) was observed to be $55 \%$ in the Mediterranean region. Maintenance of Glu-Alb (2*) and Glu-Dld (5+10) alleles is important. These alleles contribute to quality supported with Glu-Bli $(17+18)$, Glu-Blf $(13+16)$ and Glu-Blh (14+15) (Tsenov et al. 2009).

Terasawa et al. (2010) studied the genetic variation of high molecular weight gluten subunits and identified 3, 9 and 15 alleles in Glu-Al, Glu-Bl and Glu-Dl, respectively. Glu-Alc (74.4\%), Glu-Blb (76.5\%) and Glu-Dla $(81.5 \%)$ were the most frequently observed alleles. Although Glu-Dla (46.9\%) was the most frequently observed allele in Central Asia, it was lower in all the other regions except Caucasian region. A total of 83 allelic combinations were determined on Glu- 1 loci in their studies. Among the allelic combinations, Glu$A l \mathrm{c} / G l u-B l \mathrm{~b} / G l u-D l \mathrm{a}$ was the most frequently observed genotype. The frequency of this allelic combination was found to be $11.70 \%$ for Turkish cultivars. The most frequently observed allelic combination was GluBlb/Glu-Dla (22.48\%). Although western Asian, Afghanistan, and Eastern Asian wheats were exhibiting 
similar characteristics, Caucasian and Central Asian wheats differed from these three regions. As it is reported by Terasawa et al. (2009) and Lagudah et al. (1987), the most common genotypes were determined to be Glu-Alc (null), Glu-Blb (7+8), and Glu-Dla (2+12) among Western and Eastern Asian genotypes. These alleles were also found to be the highest in frequency in the present study. Those results, the results of Terasawa et al. (2009) and Lagudah et al. (1987) indicated that Glu-Alc, Glu$B l \mathrm{~b}$, and $G l u-D l$ a genotypes were dominant in regions extending from Mesopotamia, Afghanistan, and Far East to Central Asia. In Southern Asia, Glu-Alc, Glu-Bli, and Glu-Dla were the most frequently observed genotypes in a study reported by Terasawa et al. (2010). This genotype was considered to be a modified version of the typical Asian genotype in the sense that only Glu-Bli allele was replacing Glu-Blb allele. Glu-Bli allele was observed rarely in other regions of Southern Asia. This allele was also found to be very rare in the present study $(0.78 \%)$. Similarly, this allele was also rare among European endemic wheat genotypes (Gregova et al. 1999, 2006, Juha'sz et al. 2003). This is why researchers considered that Glu-Bli had appeared in Southern Asia (Terasawa et al. 2010). Glu-Bli allele was providing more firmness to dough compared to Glu-Blb allele (Payne \& Lawrence 1983, Mondal et al. 2008). Glu-Dld allele is common in Caucasian and Central Asian accessions. The high frequency of this allele in Caucasian and Central Asia is remarkable. In the same region Glu-Alb and Glu-Bla alleles have also been observed in high frequency (Terasawa et al. 2010). Glu-Dld allele is known to contribute to bread-making quality. This allele is introduced to modern wheat genotypes to increase breadmaking quality (Wrigley et al. 2015, online). The frequency of this allele was calculated to be $27.91 \%$ in the present study and the allele was observed in $50 \%$ of the wheat genotypes. This allele was also in high frequency in European wheat (Gregova et al. 1999, 2006, Juha'sz et al. 2003). However, researchers suggested the Caucasian region as the center of origin for this allele and its dispersion to other regions (Dvorak et al. 1998).

In conclusion, the quality score was found to be low in the studied genotypes (6.07). High quality score genotypes might have already been selected for breeding

\section{References}

1. Ahmad, M. 2000. Molecular marker-assisted selection of HMW glutenin alleles related to wheat bread quality by PCR-generated DNA markers. Theoretical and Applied Genetics, 101: 892-896.

2. Atanasova, D., Tsenov, N., Todorov, I. \& Ivanova, I. 2009. Glutenin composition of winter wheat varieties bred in Dobrudzha agricultural institute. Bulgarian Journal of Agricultural Science, 15: 9-19.

3. Autran, J.C. \& Feillet, P. 1985. Genetic and technological basis of protein quality for durum wheat in pasta. Proc. Protein Evaluation in Cereals and Legumes, Thessaloniki 23-24 October, 1985, Commission of the European Communities, Report EUR 10404 EN, 59-71. purposes by agricultural institutes. Relatively high quality scores in Black Sea region, where wheat breeding studies is relatively low, and in highly industrialized Marmara region support that claims. Considering the individual alleles in Glu-1 loci, the highest similarity was observed between Black Sea and the Mediterranean regions (0.9953) and the lowest similarity was between Marmara and Aegean regions (0.9472). When individual alleles and subunits are used for cluster analysis in Glu-1 loci, the Mediterranean region clustered with Black Sea and Aegean region clustered with Central Anatolia. Other regions are individually clustered and separated from these regions.

Among the studied genotypes, 4 accessions (TR32846-6, TR36948-1, TR45105 and TR63536) were determined to be reaching to the highest score (quality score 10). Of the 116 studied accessions, 6 genotypes (TR45398-4, TR48025-3, TR33264-6, TR393-5, TR52021-3 and TR45094) had the quality score of 9. To investigate new Glu-1 alleles, more landraces need to be studied. To verify new putative alleles, 2D gel electrophoresis and peptide sequencing could also be applied in addition to PCR and SDS-PAGE.

Although we detected 50 different allelic combinations among the studied accessions, we were able to calculate the quality score of 39 accessions. GluB1b/Glu-D1a with $22.48 \%$ frequency was the most frequent combination. This was followed by GluA1c/Glu-B1b/Glu-D1a, Glu-A1b/Glu-B1c/Glu-D1d and Glu-B1d/Glu-D1a allelic combinations with $11.7 \%$, $9.35 \%$ and $5.45 \%$ frequencies, respectively. The highest quality scores were observed in Glu-A1b/Glu-B1c/GluD1d (quality score 10 with $4.70 \%$ frequency) and in GluA1b/Glu-B1b/Glu-D1d (quality score 9 with $9.35 \%$ frequency) allelic combinations, respectively.

\section{Acknowledgement}

This study was supported by the Scientific Research Projects Department of Erciyes University (with the project number of FBD-3666). We are indebted to Prof. Dr. Behzad Ghaderi Sohi (Professor in English Language and Literature Department of Erciyes University) for editing the English of the manuscript.

4. Bietz, J.A., Shepherd, K.W. \& Wall, J.S. 1975. Singlekernel analysis of glutenin: Use in wheat genetics and breeding. Cereal Chemistry, 52: 513-532.

5. Branlard, G., Autran, J.C. \& Monneveux, P. 1989. High molecular weight glutenin subunits in durum wheat (Triticum durum). Theoretical and Applied Genetics, 78: 353-358.

6. Caballero, L., Martin, M.A. \& Alvarez, J.B. 2009. Genetic diversity for seed storage proteins in Lebanon and Turkey populations of wild diploid wheat (Triticum urartu Thum. Ex Gandil.). Genetic Resources and Crop Evolution, 56: 1117-1124. 
7. Carrillo, J.M., Vazquez, J.F. \& Orellana, J. 1990. Relationship between gluten strength and glutenin proteins in durum wheat cultivars. Plant Breeding, 104: 325-333.

8. De Bustos, A. \& Jouve, N. 2003. Characterization and analysis of new HMW-glutenin alleles encoded by the GluR1 locus of Secale cereale. Theoretical and Applied Genetics, 107: 74-83.

9. Dubcovsky, J. \& Dvorak, J. 2007. Genome plasticity a key factor in the success of polyploid wheat under domestication. Science, 316:1862-1866

10. Dvorak, J., Luo, M.C., Yang, Z.L. \& Zhang, H.B. 1998. The structure of the Aegilops tauschii gene pool and the evolution of hexaploid wheat. Theoretical and Applied Genetics, 97: 657-670.

11. Feldman, M. 2001. Origin of cultivated wheat. In: (Bonjean AP, Angus WJ, eds. The world wheat book: a history of wheat breeding. Paris, France: Lavoisier Publishing, 3-56).

12. Gao, X., Appelbee, M.J., Mekuria, G.T., Chalmers, K.J. \& Mather, D.E. 2012. A second overexpression allele at the Glu-B1 high-molecular-weight glutenin locus of wheat: sequence characterization and functional effects. Theoretical and Applied Genetics, 124: 333-343.

13. Gianibelli, M.C., Echaide, M., Larroque, O.R., Carrillo, J.M. \& Dubcovsky, J. 2002. Biochemical and molecular characterization of Glu-1 loci in Argentinean wheat cultivars. Euphytica, 128: 61-73.

14. Grain Sector Report, 2013. (Web page: http://www.tmo.gov.tr/upload/document/raporlar/hububats ektorraporu.pdf) (Date accessed: 01.09.2019).

15. Gregova, E., Hermuth, J., Kraic, J. \& Dotlacil, L. 1999. Protein heterogeneity in European wheat landraces and obsolete cultivars. Additional information. Genetic Resources and Crop Evolution, 46: 521-528.

16. Gregova, E., Hermuth, J., Kraic, J. \& Dotlacil, L. 2006. Protein heterogeneity in European wheat landraces and obsolete cultivars. Additional information II. Genetic Resources and Crop Evolution, 53: 867-871.

17. Gupta, R.B., Bekes, F. \& Wrigley, C.W. 1991. Prediction of physical dough properties from glutenin subunit composition in bread wheats: Correlation study. Cereal Chemistry, 68: 328-333.

18. Horvat, D., Drezner, G., Sudar, R., Simic, G., Dvojkovic, K., Spanic, V. \& Magdic, D. 2015. Distribution of wheat protein components under different genetic background and environment. Turkish Journal of Field Crops, 20(2): 150-154

19. Jiang, Q.T., Wei, Y.M., Wang, J.R., Yan, Z.H. \& Zheng, Y.L. 2006. Isolation and Sequence Analysis of HMW Glutenin Subunit 7Dy70.7 Ecoding Gene from Xinjiang Wheat (Triticum petropavlovskyi Udacz. et Migusch). Agricultural Science China, 5(2): 81-89.

20. Juhász, A., Larroque, O.R., Tamas, L., Hsam, S., Zeller, F.J., Bekes, F. \& Bedo, Z. 2003. Bankuti 1201-an old Hungarian wheat variety with special storage protein composition. Theoretical and Applied Genetics, 107: 697-704.

21. Kaplan, M., Akar, T., Kamalak, A. \& Bulut, S. 2014. Use of Diploid and Tetraploid Hulled Wheat Genotypes for Animal Feeding. Turkish Journal of Agriculture and Forestry, 38: 838-846.
22. Lafiandra, D., Tucci, G.F., Pavoni, A. \& Turchetta, T. 1997. PCR analysis of $x$ and $y$-type genes present at the complex Glu-Al locus in durum and bread wheat. Theoretical and Applied Genetics, 94: 235-240.

23. Lagudah, E.S., Flood, R.G. \& Halloran, G.M. 1987. Variation in high molecular weight glutenin subunits in landraces of hexaploid wheat from Afghanistan. Euphytica, 36: 3-9.

24. Lawrence, G.J. \& Shepherd, K.W. 1980. Variation in glutenin protein subunits of wheat. Australian Journal of Biological Sciences, 33: 221-233.

25. Li, F., Jiang, X., Wei, Y., Xia, G. \& Liu, S. 2012. Characterization of a novel type of HMW subunit of glutenin from Australopyrum retrofractum. Gene, 492: 65-70.

26. Liu, Y., Xiong, Z.Y., He, Y.G., Shewry, P.R. \& He, G.Y. 2007. Genetic diversity of HMW-GS in Chinese common wheat (Triticum aestivum L.) landraces from Hubei province. Genetic Resources and Crop Evolution, 54: 865-874.

27. Lukow, O.M., Payne, P.I. \& Tkachuk, R. 1989. The HMW glutenin subunit composition of Canadian wheat cultivars and their association with bread making quality. Journal of the Science of Food and Agriculture, 46: 451-460.

28. Ma, W., Zhang, W. \& Gale, K.R. 2003. Multiplex-PCR typing of high molecular weight glutenin alleles in wheat. Euphytica, 134: 51-60.

29. MacRitchie, F., duCros, D.L. \& Wrigley, C.W. 1990. Flour polypeptides related to wheat quality. Pp. 79-145, in: (MacRitchie, F. (Editor), Advances in Cereal Science and Technology, Vol. 10. AACC, St Paul, Minnesota, USA).

30. Margiotta, B., Colaprico, G. \& Lafiandra, D. 1987. Variation for protein components associated with quality in durum wheat lines and varieties, 314-330. Proc. 3rd Int. Workshop Gluten Proteins, 6-9 May, Budapest, Hungary.

31. McCarthy, P.L., Hansen, J.L., Zemetra, R.S. \& Berger, P.H. 2002. Rapid identification of transformed wheat using a half-seed PCR assay. BioTechniques, 32: 560-564.

32. McIntosh, R.A., Hart, G.E. \& Gale, M.D. 1994. Catalogue of gene symbols for wheat (supplement). Annual Wheat Newsletter, 40: 362-375.

33. Mishra, A., Bansal, S., Tomar, A., Khanna, V.K. \& Gang, G.K. 2009. PCR based isolation and cloning of HMW glutenin gene(s) from wheat (T. aestivum var. PBW343) and its fusion with kafirin gene promoter of sorghum. Acta Biologica Szegediensis, 53(1): 9-15.

34. Mondal, S., Tilley, M., Alviola, J.N., Waniska, R.D., Bean, S.R., Glover, K.D. \& Hays, D.B. 2008. Use of nearisogenic wheat lines to determine the glutenin composition and functionality requirements for flour tortillas. Journal of Agricultural and Food Chemistry, 56: 179-184.

35. Morgunov, A.I., Pena, R.J., Crossa, J. \& Rajaram, S. 1993. Worldwide distribution of the Glu-1 alleles in bread wheat. Journal of Genetics and Breeding, 47: 53-60.

36. Nakamura, H. 2000. The relationship between highmolecular weight glutenin subunit composition and the quality of Japanese hexaploid wheat lines. Journal of Agricultural and Food Chemistry, 48(7): 2648-2652.

37. Nakamura, H., Inazu, A. \& Hirano, H. 1999. Allelic variation in high-molecular-weight glutenin subunit Loci of Glu-1 in Japanese common wheats. Euphytica, 106: 131-138. 
38. Nei, M. 1972. Genetic distances between populations. The American Naturalist, 106: 283-292.

39. Nucia, A., Okoń, S. \& Tomczyńska-Mleko, M. 2019. Characterization of HMW glutenin subunits in European spring common wheat (Triticum aestivum L.) Genetic Resource and Crop Evolution, 66: 579-588.

40. Orth, R.A. \& Bushuk, W. 1974. Studies of glutenin. VI. Chromosomal location for subunits of glutenin of common wheat. Cereal Chemistry, 51: 118-126.

41. Payne, P.I. 1987a. Genetics of wheat storage protein and the effect of allelic variation on breadmaking quality. Annual Review of Plant Physiology, 38: 141-153.

42. Payne, P.I. 1987b. The genetical basis of bread-making quality in wheat, In: Aspects of Applied Biology. Cereal Quality Association of Applied Biologists. 15: 79-90.

43. Payne, P.I. \& Lawrence, G.J. 1983. Catalogue of alleles for the complex gene loci GluA1, GluB1, GluD1, which code for the highmolecular-weight subunits of glutenin in hexaploid wheat. Cereal Research Communications, 11: 29-35.

44. Payne, P.I., Corfield, K.G. \& Blackman, J.A. 1979. Identification of a high molecular weight subunit of glutenin whose presence correlates with breadmaking quality in wheats of related pedigree. Theoretical and Applied Genetics, 55: 153-159.

45. Payne, P.I., Holt, L.M., Jackson, E.A. \& Law, C.N. 1984. Wheat storage proteins: Their genetics and their potential for manipulation by plant breeding. Philosophical Transactions of the Royal Society B, 304: 359-371.

46. Payne, P.I., Holt, L.M. \& Law, C.N. 1981. Structural and genetical studies of the high molecular subunits of wheat glutenin. I. Allelic variation in subunits amongst varieties of wheat. Theoretical and Applied Genetics, 60: 229-236.

47. Payne, P.I., Holt, L.M. \& Lawrence, G.D. 1983. Detection of a novel high-molecular-weight subunit of glutenin in some Japanese hexaploid wheats. Journal of Cereal Science, 1: 3-8.

48. Payne, P.I., Nightingale, M.A., Krattiger, A.F. \& Holt, L.M. 1987. The relationship between HMW glutenin subunit composition and the bread-making quality of British-grown wheat varieties. Journal of the Science of Food and Agriculture, 40: 51-65.

49. Pogna, N.E., Autran, J.C., Mellini, F., Lafiandra, D. \& Feillet, P. 1990. Chromosome 1B encoded gliadins and glutenin subunits in durum wheat: Genetics and relationship to gluten strength. Journal of Cereal Science, 11: 15-34.

50. Porceddu, E., Ceoloni, C., Lafiandra, D., Tanzarella, O.A. \& Mugnozza, G.T.S. 1988. Genetic resources and plant breeding: problems and prospects, 7-22. Proc. 7th Int. Wheat Genetics Symposium, 13-19 July, Cambridge-UK.

51. Radovanovic, N. \& Cloutier, S. 2003. Gene-assisted selection for high molecular weight glutenin subunits in wheat doubled haploid breeding programs. Molecular Breeding, 12: 51-59.

52. Salmanowicz, B.P. \& Dylewicz, M. 2007. Identification and characterization of high-molecular-weight glutenin genes in Polish triticale cultivars by PCR-based DNA markers. Journal of Applied Genetics, 48(4): 347-357.
53. Shewry, P.R., Halford, N.G. \& Lafiandra, D. 2003. Genetics of wheat gluten proteins. Advances in Genetics, 49: 111-184.

54. Sultana, T., Ghafoor, A. \& Ashraf, M. 2007. Genetic variability in bread wheat (Triticum aestivum L.) of Pakistan based on polymorphism for high molecular weight glutenin subunits. Genetic Resources and Crop Evolution, 54: 1159-1165

55. Temizgul, R., Akbulut, M. \& Lafiandra, D. 2018. Genetic diversity of high-molecular-weight glutenin subunit compositions in bread wheat landraces originated from Turkey. Plant Genetic Resources, 16(1): 28-38.

56. Terasawa, Y., Takara, K., Hirano, H., Kato, K., Kawahara, T., Saskuma, T. \& Sasanuma, T. 2010. Genetic variation of high-molecular-weight glutenin subunit composition in Asian wheat. Genetic Resources and Crop Evolution, 58: 283-289.

57. Terasawa, Y., Kawahara, T., Sasakuma, T. \& Sasanuma, T. 2009. Evaluation of the genetic diversity of an Afghan wheat collection based on morphological variation, HMW glutenin subunit polymorphisms, and AFLP. Breeding Science, 59: 361-371.

58. Tok, D., Senturk-Akfirat, F., Sevinç, D., Aydin, Y. \& Altinkurt-Uncuoglu, A. 2011. Identification of genetic polymorphism and DNA methylation pattern in wheat (Triticum aestivum L.). Turkish Journal of Field Crops, 16(2): 157-165.

59. Tsenov, N., Atanasova, D., Todorov, I., Ivanova, I. \& Stoeva, I. 2009. Allelic diversity in Bulgarian winter wheat varieties based on polymorphism of glutenin subunit composition. Cereal Research Communications, 37(4): 551-558.

60. TMO, 2017. Toprak mahsulleri ofisi genel müdürlüğü. (Web page: http://www.tmo.gov.tr) (Date accessed: 10.11.2018).

61. Uthayakumaran S., Beasley, H.L., Stoddard, F.L., Keentok, M., PhanThien, N., Tanner, R.I. \& Békés, F. 2002. Synergistic and additive effects of three high molecular weight glutenin subunit loci, I. Effects on wheat dough rheology. Cereal Chemistry, 79: 294-300.

62. van Hintum, T.J.L. \& Elings, A. 1991. Assessment of glutenin and phenotypic diversity of Syrian durum wheat landraces in relation to their geographical origin. Euphytica, 55: 209-215.

63. Vavilov, N.I. 1951. The origin, variation, immunity and breeding of cultivated plants. In: Selected writing of N.I. Vavilov, translated from the Russian by K. Starr Chester. Chronica Botanica, 13: 1-366.

64. Wrigley, C.W., Bekes, F., Cavanagh, C.R. \& Bushuk, W. 2015. The gluten composition of wheat varieties and genotypes, online. (Web page: http://www.aaccnet.org/initiatives/definitions/Pages/gliadi n.aspx) (Date accessed: 10.11.2018)

65. Xu, Q., Xu, J., Liu, C.L., Chang, C., Wang, C.P., You, M.S., Li, B.Y. \& Liu, G.T. 2008. PCR-based markers for identification of HMW-GS at Glu-1Bx loci in common wheat. Cereal Science, 47: 394-398. 